\title{
OFDM Baud Rate Limitations in an Optical Heterodyne Analog Fronthaul Link using Unlocked Fibre Lasers
}

\author{
Amol Delmade \\ School of Electronics Engineering \\ Dublin City University \\ Dublin, Ireland \\ amol.delmade2@mail.dcu.ie
}

R. David Koilpillai

Department of Electrical Engineering

Indian Institute of Technology Madras

Chennai, India

koilpillai@ee.iitm.ac.in

\author{
Colm Browning \\ School of Electronics Engineering \\ Dublin City University \\ Dublin, Ireland \\ colm.browning@dcu.ie
}

Deepa Venkitesh

Department of Electrical Engineering

Indian Institute of Technology Madras

Chennai, India

deepa@ee.iitm.ac.in

\author{
Arman Farhang \\ Department of Electronics Engineering \\ Maynooth University \\ Maynooth, Ireland \\ arman.farhang@mu.ie \\ Liam P. Barry \\ School of Electronics Engineering \\ Dublin City University \\ Dublin, Ireland \\ liam.barry@dcu.ie
}

\begin{abstract}
The phase noise (PN) of a photo-generated mmwave carrier, resulting from frequency and phase fluctuations of uncorrelated laser sources, limits the performance of heterodyne/millimeter-wave analog radio-over-fibre links. This work analyzes the effect of subcarrier baud rate and frequency offset (FO) variations on the performance of a $60 \mathrm{GHz}$ OFDM signal generated using unlocked fiber lasers. Conventional digital techniques for $F O$ and $P N$ compensation, in a $25 \mathrm{~km}$ mm-wave A-RoF heterodyne system, are shown to overcome relatively large FOs and to enable the successful transmission of $\mathrm{kHz}$ range sub-carrier baud rates - in line with recent 5G recommendations.
\end{abstract}

Keywords- Millimeter Wave, Radio-over-Fiber, Optical Fronthaul, Fiber-Wireless, $5 G$

\section{INTRODUCTION}

The appetite for high speed broadband due to the successful deployment of a wide range of data intensive applications has fuelled the continuous technological advances in mobile cellular networks. Future network requirements can be fulfilled by the ultra-dense deployment of small cell antenna sites and increased signal bandwidth which can be aided by moving toward millimeter wave (mm-wave) frequencies (30-300 GHz) in conjunction with a centralized radio access network (C-RAN) architecture as envisioned in $5^{\text {th }}$ generation $(5 \mathrm{G})$ technology [1]. C-RAN advocates for the functionality split, of the base station, into centralized base band unit (C-BBU), at the central station (CS), and simpler remote radio head (RRH) unit, at the antenna site, connected by fibre - termed as fronthaul. Currently deployed common public radio interface (CPRI) fronthaul links use a digitized baseband-over-fibre (DBBoF) approach which digitizes the analog signal resulting in higher fronthaul bandwidth and complex RRH architecture. An analog radio-over-fibre (A-RoF) approach retains the inherent bandwidth efficiency of the wireless signal over the fronthaul link and simplifies the RRH site architecture by avoiding the use of expensive analog-to-digital and digitalto-analog converters [2].

This work has emanated from research supported in part by a research grant from Science Foundation Ireland (SFI) under grant number 18/SIRG/5579 and VAJRA faculty scheme of the Department of Science and Technology, Govt. of India. This work is co-funded under the European Regional Development Fund under grant numbers 13/RC/2077, $12 / \mathrm{RC} / 2276$ and $15 / \mathrm{US}-\mathrm{C} 2 \mathrm{C} / \mathrm{I} 3132$
Optical heterodyning, wherein two optical carriers with a spacing equal to the desired frequency beat on a high-speed photodetector, has been studied extensively for the generation of mm-wave carriers [3], [4]. It also facilitates the distribution of mm-wave carrier to RRH sites through compatibility with optical fronthaul. Our previous work has combined the use of A-RoF with remote heterodyning using a gain switching comb source [4]. Mm-wave phase noise, resulting from the combined linewidths of the optical tones, is shown to be a major performance limiting factor in analog RoF systems [5] due to the relatively small subcarrier baud rates/bandwidths associated with multicarrier mobile waveforms - potentially as low as $15 \mathrm{kHz}$ [11].

Previous optical heterodyne demonstrations have focused on getting more stable optical tones and low phase noise through the use of dual mode lasers [6], optical frequency combs [5], optical injection [7] or RF envelope detectors [8], [9]. These methods either require non-commercially available lasers or result in additional optical/electrical complexity in the system design. These issues have motivated this work which seeks to demonstrate A-RoF mm-wave fronthaul using 'off-the-shelf' lasers without any requirement for optical locking or coherence in the transmission system. Clearly, moving to this type of implementation introduces the critical issues of relative frequency drift of the optical carriers, and generated mmwave phase noise resulting from the heterodyning of uncorrelated sources. These performance limiting factors are addressed through digital FO and PN compensation using the 'Schmidl and Cox' (S\&C) [10] and decision directed least mean square (DD-LMS) algorithms, in a $60 \mathrm{GHz}$ orthogonal frequency division multiplexing (OFDM) A-RoF heterodyne transmission system.

We have simulated, in our previous work [5], the effect of optical linewidth variation on the performance of a 2 Mbaud subcarrier spacing multi-carrier signal. Results in [5] conclude that lasers with linewidths less than $1.5 \%$ of the subcarrier baud rate are required for the system performance to be below the forward error correction (FEC) limit employing 64-QAM modulation format on the subcarriers. The effect of FO was not considered in this simulation analysis and no extra processing was done to compensate the phase noise. For the experimental work presented in this paper we varied the baud rate of the OFDM signal, while 


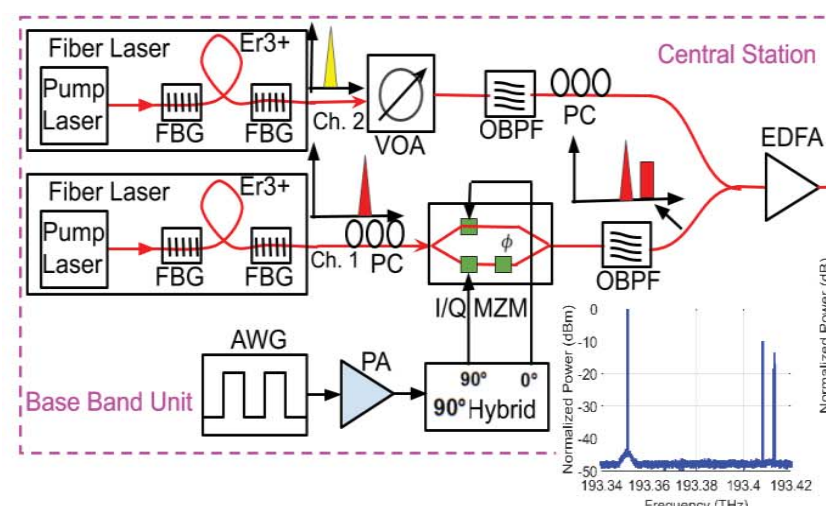

Freque

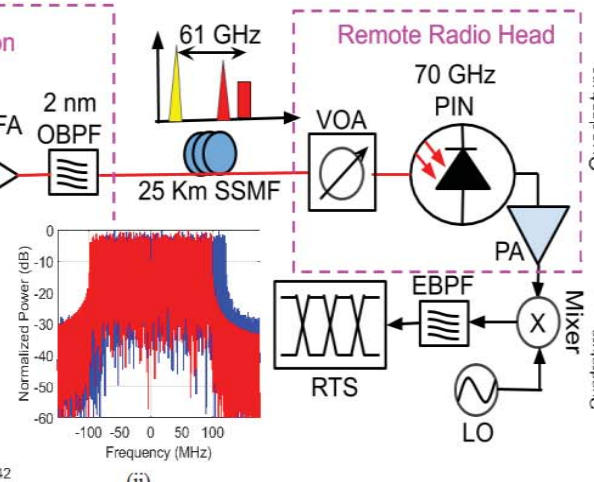

(ii)

Fig. 1: Optical heterodyne mm-wave A-RoF experimental setup including figurative optical and electrical spectra along the system path. The inset shows (i) the measured optical spectrum at the output of the transmitter, (ii) transmitted (blue) and received (red) base band spectra, constellation of a received signal (iii) without FO and PN compensation and (iv) after all DSP processes.

maintaining constant lasing conditions of two independent fiber lasers which exhibit un-correlated phase noise and a relative FO. Results presented show how the performance of a $61 \mathrm{GHz}$ OFDM signal varies as we reduce its subcarrier baud rate below $2 \mathrm{MHz}$ - with successful transmission demonstrated at baud rates as low as $125 \mathrm{kHz}$ when FO/PN compensation is used.

The fiber lasers used in this experimental work exhibit a maximum relative drift of $\sim 6 \mathrm{MHz}$ over several signal captures, and results show that it is completely compensated by the digital algorithms used. To extend the testing of the receiver FO compensation in this heterodyne context, performance is also evaluated when an additional FO is introduced in the system by varying the receiver mm-wave local oscillator (LO) frequency. The novel combination of un-locked, commercially available small form factor lasers with well-established digital processing in a heterodyne ARoF system represents a robust and deployable system for future mm-wave fronthaul networks.

\section{EXPERIMENTAL DETAILS}

\section{A. Schematics Details}

The schematic of the experimental testbed is shown in Fig.1. Two low noise fibre lasers each with output power of $+10.5 \mathrm{dBm}$ and linewidth of $\sim 1 \mathrm{kHz}$ are used at the CS. The carrier in Ch.1 (red carrier in Fig. 1) was modulated with $\sim 200 \mathrm{MHz}$ bandwidth variable subcarrier baud rate OFDM signal, at an intermediate frequency (IF) of $5 \mathrm{GHz}$. The OFDM signal was generated in the electrical domain using an arbitrary waveform generator (AWG) operating at 20 $\mathrm{GSa} / \mathrm{s}$. More details of the OFDM signal parameters for different baud rates are given in subsection B. Optical single sideband (O-SSB) modulation was performed using an electrical $90^{\circ}$ hybrid coupler and I/Q Mach Zehnder Modulator (MZM). The OSSB modulated carrier was combined with the un-modulated carrier from $\mathrm{Ch}$. 2 (yellow carrier in Fig. 1), which is $56 \mathrm{GHz}$ away from the Ch. 1 carrier, and transmitted through $25 \mathrm{~km}$ of standard single mode fibre (SSMF) after amplification by an Erbium doped fibre amplifier (EDFA). A variable optical attenuator (VOA) was used in Ch. 2 to equalize the optical power differences (due to I/Q MZM insertion loss and OSSB modulation loss in Ch. 1) between the two optical paths at the transmitter. Inset (i) in Fig. 1 shows the spectra of the signal after a $2 \mathrm{~nm}$

optical band pass filter (OBPF), which is used for filtering out-of-band amplified spontaneous emission (ASE) noise.

Beating of the un-modulated carrier (yellow carrier of $\mathrm{Ch} 2$ in Fig. 1) and data sideband (red data of Ch1 in Fig. 1) on a $70 \mathrm{GHz}$ PIN photo-detector at the 'RRH' site generates the OFDM data band at $61 \mathrm{GHz}$, which can be directly transmitted to the wireless user using antenna elements after amplification. A VOA was used to control the power falling on the PD. It is worth mentioning that wireless transmission of the mm-wave signal was not carried out in this experiment. The $61 \mathrm{GHz}$ OFDM signal was frequency down converted to the IF band using a $56 \mathrm{GHz}$ external $\mathrm{LO}$ and mixer. The frequency of this LO was intentionally varied in order to introduce additional FOs. This allowed the capabilities of the S\&C FO compensation algorithm to be tested for FOs beyond those caused by the relative drift of the lasers alone $(\sim 6 \mathrm{MHz})$. A real time oscilloscope (RTS) operating at $50 \mathrm{GSa} / \mathrm{s}$ was used to capture this IF data. Offline digital signal processing (DSP) algorithms (described below) are applied to the captured OFDM signal in Matlab before performance is evaluated through bit error rate $(\mathrm{BER})$ and error vector magnitude $(\mathrm{EVM})$ calculations.

\section{B. Baud Rate Specifications}

Variable subcarrier baud rate OFDM signals were generated by changing the IFFT size and keeping the baseband sampling rate constant. To keep the signal bandwidth constant, the number of 64 QAM data modulated subcarriers were also increased in proportion to the IFFT size. 100 subcarriers out of 256 were modulated by 64 QAM data to get a baud rate of $\sim 2 \mathrm{MHz}$ resulting in a raw data rate of $1.2 \mathrm{~Gb} / \mathrm{s}$. Both IFFT size and the number of data subcarriers were increased by a factor of 2 with respect to previous values to reduce the baud rate by a factor of 2 each time. OFDM signals with subcarrier baud rate values of 2 $\mathrm{MHz}, 1 \mathrm{MHz}, 500 \mathrm{kHz}, 250 \mathrm{kHz}, 125 \mathrm{kHz}$ and $62.5 \mathrm{kHz}$ were generated, and their performance was analyzed upon transmission over the $25 \mathrm{~km}$ SSMF based mm-wave A-RoF heterodyne system. These signals were up-sampled and digitally mixed with a $5 \mathrm{GHz}$ IF carrier before being loaded to the AWG.

\section{DSP for FO and PN compensation}

Uncorrelated fluctuations in the carrier frequencies (yellow and red carriers in Fig. 1) of the two free running fibre lasers changes the frequency spacing between them. 
This results in different IF center frequency of the captured signal leading to frequencies offset as shown in the frequency down converted signal spectra (inset (ii) in Fig. 1). As explained previously, these random fluctuations result in the random relative phase variations between LO and $61 \mathrm{GHz}$ generated mm-wave signal giving rise to phase noise in the system. The FO and PN need to be compensated for the reliable detection of the transmitted data.

The captured signal from the RTS was processed for FO and $\mathrm{PN}$ compensation after resampling and frequency down conversion to baseband. Direct ODFM demodulation of this frequency down-converted baseband signal, without application of any compensation algorithms, results in the constellation as shown in the inset (iii) of Fig. 1. It is impossible to retrieve the data due to the FO and PN effects without the application of the DSP algorithms. Searching for a special symbol with two identical halves, i.e. preamble as defined in the S\&C algorithm [10], in the frequency down converted signal helps with the timing synchronization along with FO estimation. This preamble was transmitted at the start of the OFDM data signal for each baud rate case. Using the preamble, FOs that were a fraction of the baud rate (subcarrier bandwidth) were corrected by estimating the phase shift caused by the received signal FO [10]. The remaining integer (subcarrier index) FO was detected by cross correlation of the transmitted and received preamble, instead of using a second preamble as described in S\&C algorithm. The detected data is counter rotated in the frequency domain by a number of samples equal to the integer FO to completely correct for the FO. To determine the amount of FO that can be compensated by this algorithm, we have introduced an additional FO by varying the mm-wave LO in $20 \mathrm{MHz}$ steps for some of the baud rates, and the results are discussed in section III.

Phase correction is applied by employing the least mean square phase tracking which compares the FO compensated data with the standard 64-QAM constellation points. As this technique is blind and depends on the squared error with the ideal constellation points, it leads to a phase ambiguity resulting in a fixed phase shift in the symbols. This fixed phase was estimated by using pilots placed along the subcarriers of the OFDM data. Inset (iv) in Fig. 1 shows the constellation of the OFDM demodulated signal after employing all necessary digital post-processing.

\section{RESULTS AND DISCUSSION}

EVM performance of the transmission system described is measured with variable baud rates and FO under the influence of uncorrelated fluctuations of the two optical carriers used in this experiment. Transmitted data can be correctly retrieved by applying the DSP compensation algorithms described earlier, as can be seen from the constellation diagrams transformation from the one shown in inset (iii) to that in inset (iv) after the relevant DSP is implemented. This constellation diagram corresponds to the $2 \mathrm{MHz}$ subcarrier baud rate OFDM signal and represents a received EVM of $4.76 \%$ with measured BER to be $3.13 \times 10^{-5}$ for a received optical power of $-2 \mathrm{dBm}$. We have also observed that there is no penalty due to $25 \mathrm{~km}$ fibre transmission. The excellent performance obtained for this mm-wave A-RoF link is attributed to the good quality of fibre lasers with SMSR $>50 \mathrm{~dB}$ and RIN better than -140 $\mathrm{dBc} / \mathrm{Hz}$.

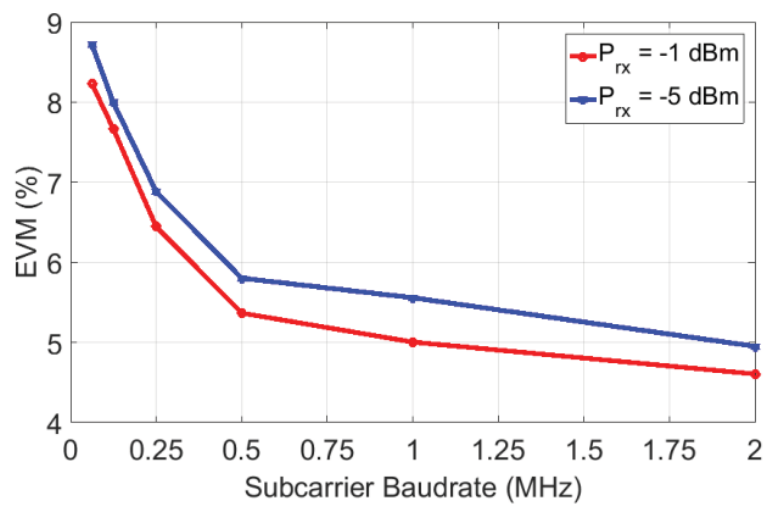

Fig.2: EVM versus subcarrier baud rate for DSP compensation based $61 \mathrm{GHz}$ OFDM mm-wave A-RoF system.

\section{A. Performance with variable baud rates}

Variable baud rate signals were generated and transmitted through the $25 \mathrm{~km}$ SSMF mm-wave A-RoF system and performance was analyzed at two different power levels falling on the PD. Fig. 2 shows the EVM performance of the $200 \mathrm{MHz}$ bandwidth OFDM signal with variable subcarrier baud rates for received powers of $-1 \mathrm{dBm}$ and $-5 \mathrm{dBm}$. The figure shows that for the implemented system, performance degrades as the subcarrier baud rate reduces. The phase noise of the generated mm-wave carrier does not significantly affect the performance of higher baud rate signals, but as the subcarrier baud rate reduces, the phase noise on the RF beat signal (due to combined optical phase noise of the fiber lasers) starts to degrade the system performance. Performance goes above the FEC EVM limit of $8 \%$, for 64-QAM modulated data, at the subcarrier spacing of $62.5 \mathrm{kHz}$ and below. Performance degradation $\leq$ $0.5 \%$ in the EVM is observed when the power falling on the $\mathrm{PD}$ is changed from $-1 \mathrm{dBm}$ to $-5 \mathrm{dBm}$.

We can infer that for successful transmission of the 62.5 $\mathrm{kHz}$ subcarrier baud rate OFDM signal, optical sources with a linewidth of $<1 \mathrm{kHz}$ will be required. The linewidth $(\sim 1$ $\mathrm{kHz}$ ) of the lasers used in this experiment corresponds to $\sim 1.5 \%$ of the lowest subcarrier baud rate $(62.5 \mathrm{kHz})$ used. This is in agreement with the simulation results presented in our previous work [5]. It is important to note that the simulations presented in [5] do not consider the FO effect but our DSP compensations techniques were able to overcome the FO effect and achieve close to simulation performance. Lasers with linewidths of $\sim 225 \mathrm{~Hz}$ will be required for the successful transmission of 64-QAM multicarrier signals with the lowest subcarrier baud rates of 15 $\mathrm{kHz}$ suggested by $5 \mathrm{G}$ NR physical layer specifications [11], over a mm-wave A-RoF heterodyne system.

\section{B. Performance with $F O$ variations}

The fibre lasers used in the experimental setup drift by $\sim 6$ $\mathrm{MHz}$ over several captures. The DSP used for compensation of the FO and PN operate successfully over this range as seen from the constellation diagram of inset (iv) in Fig. 1 and the EVM performance of Fig. 2. This does not completely test the capabilities of the FO compensation algorithm. For this purpose we introduced the FO in the signal by changing the frequency of the RF (mm-wave) LO at the RRH site. Fig. 3 shows the EVM performance of the system with $\mathrm{FO}$ variations for three different subcarrier baud 


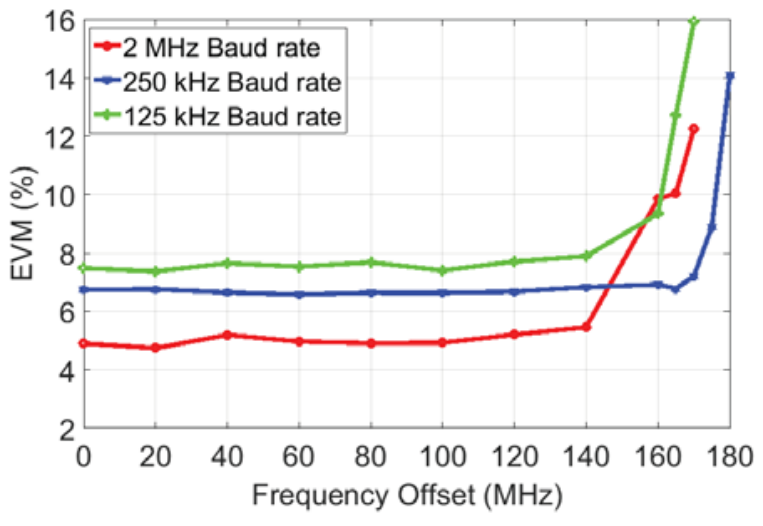

Fig.3: EVM versus LO induced frequency offset performance for DSP compensation based $61 \mathrm{GHz}$ OFDM A-RoF system

rates at a received power of $-1 \mathrm{dBm}$. Results shows that an LO induced frequency offset of up to $140 \mathrm{MHz}$ can be compensated digitally without any significant degradation in the system performance - and it is independent of the signal's subcarrier baud rate. Degradation in the performance can be observed from this graph for FO's beyond $140 \mathrm{MHz}$, but FO's below this level should be easily achievable through the use of a number of standard laser and thermoelectric cooler technologies. Performance degradation for FOs beyond $140 \mathrm{MHz}$ can be attributed to the limitations of the S\&C algorithm [10].

\section{CONCLUSION}

Remote optical heterodyning can be used in future wireless systems for the generation and distribution of mmwave analog signals over optical fronthaul networks. Low linewidth lasers in conjunction with digital processing, for compensating frequency drift and phase offset between the optical carriers, are of paramount importance for facilitating the transmission of small baud rate multicarrier signals, as envisioned in $5 \mathrm{G}$ technology. The results presented here demonstrate the successful transmission of $61 \mathrm{GHz}$ OFDM signals with subcarrier baud rates of $125 \mathrm{kHz}$ and higher, when $\mathrm{FO} / \mathrm{PN}$ compensation is used, over a mm-wave A-RoF heterodyne system using unlocked fiber lasers. In the proposed system, frequency offsets of around $140 \mathrm{MHz}$ and less can be compensated by using digital processing without any significant performance degradation. This paves the way for the utilization of 'off-the-shelf' fibre lasers coupled with well-established digital processing for the transmission of the low baud rates multicarrier signals over spectrally efficient mm-wave A-RoF fronthaul networks.

\section{REFERENCES}

[1] J. G. Andrewa, S. Buzzi, W. Chop, S. V. Hanly, A. Lozano, A. C. K. Soong, and J. C. Zhang, "What will 5G Be?," IEEE Journal on Selected Areas in Communications, vol. 32, no. 6, pp. 1065-1082, June 2014

[2] A. Delmade, C. Browning, A. Farhang, N. Marchetti, L. E. Doyle, R. D. Koilpillai, L. P. Barry and Deepa Venkitesh, "Performance Analysis of Analog IF Over Fiber Fronthaul Link With 4G and 5G Coexistance," Journal of Optical Communications and Networking, 10 (3), pp. 174-182, January 2018.

[3] Li Fan, Guangqiong Xia, Jianjun Chen, Xi Tang, Qing Liang, and Zhengmao $\mathrm{Wu}$, "High-purity $60 \mathrm{GHz}$ band millimeter-wave generation based on optically injected semiconductor laser under subharmonic microwave modulation," Opt. Express, 2016,24, pp. $18252-18265$

[4] C. Browning, E. P. Martin, A. Farhang and L. P. Barry, "60 GHz 5G Radio-over-fibre using UF-OFDM with optical heterodyning," IEEE Photonics Technology Letters, 2017, 29 (23), pp. 2059-2062

[5] C. Browning, H. H. Elwan, E. P. Martin, Sean O'Duill, J. Poette, P. Sheridan, A. Farhang, B. Carbon and L. P. Barry, "Gain-switched optical frequency combs for future mobile radio-over-fibre millimeter-wave systems," Journal of Lightwave Technology,2018, 6 (19), pp. 4602-4610

[6] M.C. Gross, P. Callahan, T.R. Clark, D. Novak, R.B. Waterhouse and M.L. Dennis, "Tunable millimeter-wave frequency synthesis up to $100 \mathrm{GHz}$ by dual-wavelength Brillouin fibre laser," Opt. Express, 2010,18, pp. 13321-13330

[7] Li Fan, Guangqiong Xia, Jianjun Chen, Xi Tang, Qing Liang, and Zhengmao $\mathrm{Wu}$, "High-purity $60 \mathrm{GHz}$ band millimeter-wave generation based on optically injected semiconductor laser under subharmonic microwave modulation," Opt. Express, 2016,24, pp. 18252-18265

[8] M. F. Hermelo, Po-Tsung Shih, M. Steeg, A. Ng'oma, and A. Stöhr, "Spectral efficient 64-QAM-OFDM terahertz communication link," Opt. Express, 2017,25, pp. 19360-19370

[9] T. Xu, S. Mikroulis, J.E. Mitchell, and I. Darwazeh, "Bandwidth compressed waveform for $60-\mathrm{GHz}$ millimeter-wave radio over fibre Experiment," Journal of Lightwave Technology,34, 3458-3465 (2016)

[10] T.M. Schmidl and C. Cox, "Robust Frequency and Timing Synchronization for OFDM," IEEE Trans. On Communications, 1997, 45 (12), pp. 1613-1621

[11] Javier Campos, "Understanding the 5G NR Physical Layer," Keysight Technologies release, November 2017. 